\title{
Validity of the Brazilian version of the Neuropsychiatric Inventory Questionnaire (NPI-Q)
}

\author{
Validação da versão brasileira do Questionário do Inventário Neuropsiquiátrico (Q-INP) \\ Ana Luiza Camozzato ${ }^{1,4}$, Claudia Godinho1,2, Renata Kochhann¹, Graziela Massochini², \\ Marcia Lorena Chaves ${ }^{1,2,3}$
}

\begin{abstract}
The NPI-Q (Neuropsychiatry Inventory-Questionnaire) was developed to facilitate the evaluation of neuropsychiatric symptoms. This study evaluated the internal consistency, the test-retest reliability of the Brazilian NPI-Q version and its convergent validity with the original NPI. Method: The NPI-Q and the NPI were administered to 64 caregivers of dementia patients. Thirteen informants were asked to complete a second NPI-Q form. Results: The internal consistency of the Brazilian NPI-Q version was 0.67 for the severity scale and 0.81 for the distress scale. The test-retest reliability of the total NPI-Q severity and the distress scales were 0.97 and 0.92 , respectively $(p<0.001)$. There were significant correlations between the total NPI-Q severity score and the NPI ( $r=0.75)$ and between the total NPI-Q distress score and the total NPI standard distress $(r=0.74)$. Conclusion: The Brazilian NPI-Q version showed evidence of good psychometric properties and can be used in general clinical practice.
\end{abstract}

Keywords: Brazilian NPI-Q version, validation study, neuropsychiatric symptoms, dementia.

\begin{abstract}
RESUMO
O Q-INP (Questionário do Inventário Neuropsiquiátrico) foi desenvolvido para facilitar a avaliação dos sintomas neuropsiquiátricos. Este estudo avaliou a consistência interna, confiabilidade teste-reteste e validade convergente da versão brasileira do Q-INP com o INP (Inventário Neuropsiquiátrico). Método: Sessenta e quatro cuidadores de pacientes com demência responderam ambas as escalas. 0 NPI-Q foi reaplicado em 13 informantes. Resultados: A consistência interna da versão brasileira do Q-INP foi 0,67 para a escala de gravidade e 0,81 para escala de desgaste. A confiabilidade teste-reteste da escala de gravidade foi 0,97 e 0,92 para a escala de desgaste $(p<0,001)$. Houve correlação significativa entre o escore de gravidade do Q-INP e INP $(r=0,75)$ e entre os escores de desgaste destas escalas $(r=0,74)$. Conclusão: A versão brasileira do Q-INP mostrou evidências de boas propriedades psicométricas e pode ser usado na prática clínica geral.
\end{abstract}

Palavras-chave: versão brasileira do Q-INP, estudo de validação, sintomas neuropsiquiátricos, demência.

The recognition of neuropsychiatric symptoms in dementia is relevant since they are frequent, distressing for patients and for caregivers ${ }^{2}$, and characterize a frequent cause of institutionalization ${ }^{3}$. Among the scales used to evaluate these symptoms, the Neuropsychiatric Inventory (NPI), an informant-based interview ${ }^{4}$, is one of the most widely used instruments in clinical research studies ${ }^{5}$. However, it takes long time to be completed, making difficult its use in the general practice setting. In this scenario, a brief NPI version, the Neuropsychiatric Inventory Questionnaire
(NPI-Q), was developed to facilitate the evaluation of neuropsychiatric symptoms. The NPI-Q derives from NPI, assessing the same 12 NPI symptom domains and the caregiver's distress produced by these domains. It is a self-administered questionnaire, in opposition to the original NPI which is based on informant interview; and it assesses only symptom severity, instead of severity and frequency of the symptoms as measured in the NPI. In addition, it is usually completed in 5 minutes or less. It showed adequate test-retest reliability ( $\mathrm{r}=0.80, \mathrm{p}=0.001)$ and convergent validity with the NPI

${ }^{1}$ Departamento de Demência Clínica, Serviço de Neurologia, Hospital de Clínicas de Porto Alegre, Porto Alegre RS, Brazil;

${ }^{2}$ Faculdade de Medicina, Universidade Federal do Rio Grande do Sul, Porto Alegre RS, Brazil;

${ }^{3}$ Departamento de Clínica Médica, Faculdade de Medicina, Universidade Federal do Rio Grande do Sul, Porto Alegre RS, Brazil;

${ }^{4}$ Universidade Federal de Ciências da Saúde de Porto Alegre, Porto Alegre RS, Brazil.

Correspondence: Ana Luiza Camozzato; Rua Ramiro Barcelos, 2350 / sala 2040; 90035-091 Porto Alegre RS, Brasil; E-mail: anacamoz@gmail.com

Conflict of interest: There is no conflict of interest to declare.

Received 14 July 2014; Received 04 September 2014; Accepted 24 September 2014. 
regarding total and individual symptom domain scores and caregiver distress ratings $(\mathrm{r}=0.92, \mathrm{p}=0.001)$. The correlation between NPI and NPI-Q total symptom scores was significant $(\mathrm{p}=0.001)$ for any dementia severity $(\mathrm{r}=0.91)$, being greater in subjects with more severe dementia $(r=0.95)$. Both NPI and NPI-Q total scores showed weak, but significant, correlations with Mini Mental State Examination (MMSE) scores in the lower MMSE group $(r=0.44)$, while these associations were not observed in the higher MMSE group 6 .

The NPI-Q has been already validated for Spanish, Japanese and Dutch languages ${ }^{7,8,9}$. The NPI-Q Spanish version showed strong test-retest reliability for total symptom scale and for distress scale, besides convergent validity with NPI total symptom $(\mathrm{r}=0.879)$ and with NPI distress $(\mathrm{r}=0.92)^{7}$. The NPI-Q Japanese version also showed strong correlation with NPI for both total NPI-Q severity score $(\mathrm{r}=0.77, \mathrm{p}<0.01)$ and distress score $(\mathrm{r}=0.80, \mathrm{p}<0.01)$. It also showed high and significant test-retest reliability for severity and distress score ${ }^{8}$. The depressive symptoms measured by the NPI-Q Dutch version showed a moderate correlation with the Geriatric Depression Scale (GDS) .

The aim of this study was to evaluate the internal consistency, the test-retest reliability of the Brazilian NPI-Q version and its convergent validity with the NPI. The effect of demographic and clinical data - severity of dementia - on NPI-Q, as well as the impact of sex, age and educational level of caregivers on this scale was also evaluated. Our hypothesis was that the NPI-Q Brazilian version presents psychometric properties similar to those observed in other languages.

\section{METHOD}

A cross-sectional investigation was carried out in a sample of 64 caregivers of patients with dementia due Alzheimer's disease (AD) and vascular dementia (VD) consecutively selected from the Dementia Clinic of Hospital de Clinicas de Porto Alegre, Brazil. The $\mathrm{AD}$ dementia diagnosis was ascertained by the DSM-V ${ }^{10}$ and the Alzheimer Association criteria ${ }^{11}$. Vascular dementia was diagnosed with the DSM-V criteria for Major Vascular Neurocognitive Disorder $^{10}$. The Mini Mental State Examination (MMSE) $)^{12,13}$ and the Clinical Dementia Rating scale (CDR) were applied $^{14,15,16}$ to evaluate the severity of dementia. Demographic data from the outpatients and from caregiver informants were collected.

The NPI- $Q^{6}$ is a self-administered scale derived from the NPI standard that evaluates the same 12-symptom domains (delusions, hallucinations, agitation/aggression, dysphoria/depression, anxiety, euphoria/elation, apathy/ indifference, disinhibition, irritability/lability, aberrant motor behaviors, nighttime behavioral disturbances, and appetite/eating disturbances). Each domain is assessed by a written screening question that assesses presence and severity of the core symptom manifestations over the past month. All screening questions are in the 'yes/no' format and severity is rated as 'mild' $=1$, 'moderate' $=2$ and 'severe' $=3$. The total NPI-Q severity score ranges from 0 to 36 . In addition, the NPI-Q assesses the primary caregiver distress as in the NPI. The distress scale is rated with 6point level questions, ranging from "not emotionally stressful" to "extremely stressful", and the total NPI-Q distress score ranges from 0 to 60 .

The original English version of the NPI-Q was translated by the authors to Brazilian Portuguese. Further, the Brazilian version was independently back translated into English. Final adaptations were performed to warrant cultural and educational comprehension.

This Brazilian NPI-Q version was administered to nonprofessional caregivers who were fully aware of the patient's behavior since they spent at least three hours with the patient in a daily basis. The Brazilian version of the standard $\mathrm{NPI}^{17}$ was applied to measure the NPI-Q concurrent validity. In order to avoid biased responses in the NPI-Q by the previous application of NPI interview, the Brazilian version of the NPI-Q was first applied. The clinician who carried out the NPI interview was blinded to the results of the NPI-Q. This clinician was a member of the research team previously trained to apply the NPI. Thirteen informants (25\% of the sample) were asked to complete a further NPI-Q form upon arriving home in the same day. A member from the research team called them in the next morning to check the answers. This procedure was made to evaluate the test-retest reliability.

\section{Statistical analysis}

Descriptive analyses were previously performed. The normality of data was assessed by the Kolmogorov-Smirnov test and parametric or non-parametric statistics were carried out according to the variables distribution. The Cronbach's alpha test was used to verify the NPI-Q internal consistency. The Pearson correlation test was used to evaluate the test-retest NPI-Q reliability and to assess the bivariate correlations between NPI-Q and NPI standard (total and single item scores), as well to evaluate the correlations between NPI-Q and NPI standard stratified by dementia severity.

The Pearson correlation test was used to evaluate correlation between CDR, MMSE, age and education with the NPI-Q. The association of sex with the NPI-Q was evaluated by Chi-square association test.

The absolute and the relative difference in individual symptom frequency across NPI-Q and NPI were described.

The study was approved by the Ethics Committee for Medical Research at Hospital de Clínicas de Porto Alegre. All participants and their proxies signed an informed consent. 
Table 1. Correlations coefficients among NPI-Q, NPI, and dementia severity.

\begin{tabular}{lccc}
\hline Variable & All subjects* $(\mathrm{N}=64)$ & Mild dementia* $\left.^{*} \mathrm{~N}=31\right)$ & Moderate to severe dementia* $(\mathrm{N}=33)$ \\
\hline NPI-Q severity to NPI severity & 0.755 & 0.741 & 0.724 \\
NPI-Q severity to NPI total (FXS) & 0.705 & 0.675 & 0.692 \\
NPI-Q distress to NPI distress & 0.739 & 0.723 & 0.713 \\
NPI-Q severity to NPI-Q distress & 0.913 & 0.905 & 0.906
\end{tabular}

*Pearson correlation coefficient ( $r$ ) with $p<0.001$. NPI: Neuropsychiatric inventory; NPI-Q: NPI Questionnaire; F x S: Frequency versus severity.

\section{RESULTS}

Sixty-four non-professional caregivers of $\mathrm{AD}$ or $\mathrm{VD}$ patients answered the research protocol. The sample was composed of 57 (89\%) women. Of the 64 participants, 25 were spouses, 27 were children, and 12 had other familial relationship with the patient. Forty-eight (67\%) informants lived with the patient. The mean age of caregivers was 52.5 (standard devistion $(\mathrm{SD})=14.31$ ) and education varied from 1 to 19 years of study $(10.41 \pm 4.22$, mean $\pm \mathrm{SD})$.

The mean age of patients was $75.9(\mathrm{SD}=9.6)$, and 40 $(62 \%)$ were female. Education varied from illiteracy (zero years) to fifteen years of schooling $(4.5 \pm 3.5$, mean \pm SD). Thirty-one patients were classified as mild dementia $(\mathrm{CDR}=1)$ and 33 as moderate to severe dementia $(\mathrm{CDR}=2$ and CDR $=3)$. The mean $( \pm$ SD) MMSE score was $14.84( \pm 5.46)$.

The internal consistency of the severity scale of the Brazilian NPI-Q version was 0.67 and it was 0.81 for the distress scale. The reliability (test-retest correlation) of the total NPI-Q severity and the distress scales were 0.97 and 0.92 , respectively ( $\mathrm{p}<0.001$ for both).

The correlations between the total NPI-Q severity score and the NPI standard (total severity and total score-sum of frequency $\mathrm{x}$ severity ratings for all domains) were significant and moderate, as well the correlation between the total NPI-Q distress score and the total NPI standard distress score. These correlations coefficients were also significant and moderate when the sample was stratified by dementia severity. A stronger correlation was observed between total NPI-Q severity and total NPI-Q distress score (Table 1).
The majority of the correlations between each item of NPI-Q and NPI were statistically significant (either mild or moderate), with the exception of the severity of motor aberrant behavior and appetite/eating disturbances domains (Table 2).

The total MMSE score did not show significant correlation with NPI-Q severity score $(\mathrm{r}=-0.151, \mathrm{p}=0.235)$. The NPI-Q severity score did not differ among CDR categories $(\mathrm{F}=1,720, \mathrm{p}=0.173$, one-way ANOVA). Patients age and education did not correlate with NPI-Q severity $(\mathrm{r}=-0.145$, $p=0.331$ and $r=-0.057, p=0.671$, respectively). Men showed significantly higher scores on NPI-Q ( $\mathrm{p}=0.024$, Student $t$-test).

Among sex, age and education of caregivers, only education was significantly inversely correlated with total NPI-Q severity $(r=-0.340, p=0.007)$ and with NPI-Q distress score $(\mathrm{r}=-0.320, \mathrm{p}=0.012)$.

Table 3 shows the absolute and the relative values of individual NPI-Q and NPI frequency of symptoms and the difference between scales of the number of symptoms reported for a given domain. The frequency of all domains was higher when assessed with the NPI-Q and the average difference between scales of the number of reported symptoms was 15\% (mean absolute difference).

\section{DISCUSSION}

The present study aimed to evaluate the psychometric properties of the Brazilian NPI-Q version. This scale fulfils a gap in the evaluation of neuropsychiatric symptoms of

Table 2. Item correlation coefficients among NPI-Q and NPI scale.

\begin{tabular}{|c|c|c|c|}
\hline Subscale & NPI-Q severity to NPI-Severity & NPI-Q severity to NPI-Total $(F \times S)$ & NPI-Q Distress to NPI Distress \\
\hline Delusions & $0.697 \star$ & $0.663^{*}$ & $0.592 *$ \\
\hline Hallucinations & $0.662^{\star}$ & $0.564^{\star}$ & $0.627 *$ \\
\hline Agitation/Aggression & $0.366^{* *}$ & $0.437 * *$ & $0.537 *$ \\
\hline Dysphoria/Depression & $0.538 *$ & $0.567 *$ & $0.549 *$ \\
\hline Anxiety & $0.375^{\star}$ & $0.403 *$ & $0.304 * *$ \\
\hline Euphoria/Elation & $0.386 * *$ & $0.337 * *$ & $0.426 * *$ \\
\hline Apathy/Indifference & $0.685^{\star}$ & $0.592^{\star}$ & $0.680 *$ \\
\hline Disinhibition & $0.400^{*}$ & $0.287 * \star$ & $0.419 *$ \\
\hline Irritability/Lability & $0.447 *$ & $0.445^{\star}$ & $0.500^{*}$ \\
\hline Aberrant Motor & 0.203 (NS) & 0.179 (NS) & $0.536^{*}$ \\
\hline Nighttime Disturbances & $0.478^{*}$ & $0.441 *$ & $0.435^{\star}$ \\
\hline Appetite/Eating disturbances & 0.103 (NS) & 0.134 (NS) & $0.211(\mathrm{NS})$ \\
\hline
\end{tabular}

*Pearson correlation coefficient ( $r$ ) with $p<0.001$; **Pearson correlation coefficient ( $r$ ) with $p<0.05$. NS: Non significant; NPI: Neuropsychiatric inventory; NPI-Q: NPI Questionnaire; F x S: Frequency versus severity. 


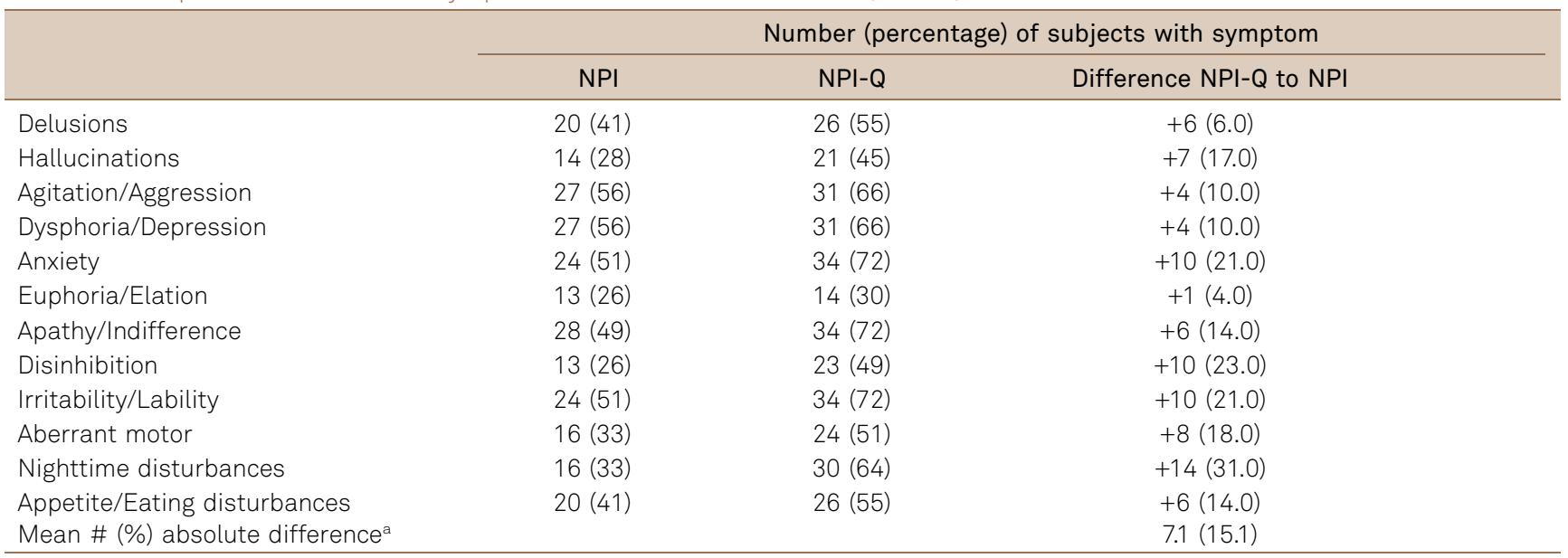

${ }^{a}$ Mean absolute difference reflects the average difference between scales of the number of reported symptoms for each domain. NPI: Neuropsychiatric inventory; NPI-Q: NPI questionnaire.

dementia, because it is a brief and feasible screening tool, of easy application in clinical settings, and allows the recognition and management of these frequent and distressing symptoms. It measures the severity of NP symptoms and the caregiver distress related to them. The Brazilian NPI-Q version showed adequate internal consistency and test-retest reliability. The scores of symptoms domain and the caregiver distress ratings were equivalent to those assessed by the NPI, demonstrating good convergent valid. The internal consistency was moderate for severity scale and strong for distress scale. Its test-retest was strong and significant; ensuring its stability over the time. Although the correlation with NPI was statistically significant and moderate for both scales and across all dementia severity stages, these correlation coefficients were slightly smaller than those observed in the other NPI-Q versions ${ }^{6,7}$. It is possible that the wide range of caregiver's educational attainment (ranging from 1 to 19 years), might have influenced our results. Informants with lower education could have some difficulty to understand and to answer the questionnaire.

The neuropsychiatric symptom frequency was higher in the Brazilian version of the NPI-Q than in the NPI. Kaufer and colleagues found similar result, however the difference between prevalence assessed by the two scales was greater in our study ( $15 \%$ overall). This result could be partially explained by the expected higher sensitivity and lower specificity of the screening questions. In fact, a rate of $5 \%$ of false positive in the NPI screening questions had already been showed 4 . Furthermore, some screening questions of NPI-Q are more open question then those from NPI, i.e., are less detailed and give a few number of examples. This difference could have produced NPI-Q false-positive answers. For example, the nighttime behavior showed the highest difference of frequency between NPI-Q and NPI, with higher NPI-Q score. The screening question in the NPI-Q is
"Does the patient awaken you during the night, rise too early in the morning, or take excessive naps during the day?", while the screening question in the NPI is "Does the patient have difficulty sleeping (do not count as present if the patient simply gets up once or twice per night only to go to the bathroom and falls back asleep immediately)? Is he/she up at night? Does he/she wander at night, get dressed, or disturb your sleep?" Other possible explanations for this result could be cultural characteristics, i.e. informants with more "permissiveness" to be complaining; and socio-economic reasons, such as higher caregiver burden due to the scarce support sources in developing countries. However the study design did not allow for testing these hypotheses.

Since the NPI-Q is an informant-based instrument, we think it was important to examine the association of this scale with age, education and sex of caregivers. Among these variables, only education was inversely correlated with the NPI-Q severity and NPI-Q distress scores. Although this correlation can only be a random finding, we can hypothesize that the lower caregiver's education could impair the comprehension of the scale leading to higher rates of falsepositive answers. This issue was not the focus of the present study, but further studies with properly design should address this relevant question. Other caregiver's characteristics such as affiliation degree with the patient, years as caregiver and to receive payment could impact the perception of the patient and its behavior and also should be investigated.

Finally, the Brazilian NPI-Q version demonstrated evidence of good psychometric properties. Therefore, the NPI-Q can be used in the clinical practice or research settings as a comprehensive, practical, reliable and brief instrument to measure neuropsychiatric symptoms in subjects with dementia and to assess the related emotional stress of primary caregivers. 
1. Steinberg $\mathrm{M}$, Shao $\mathrm{H}$, Zandi $\mathrm{P}$, et al. Point and 5-year period prevalence of neuropsychiatric symptoms in dementia: the Cache County Study. Int J Geriatr Psychiatry 2008;23:170-177.

2. Robinson KM, Adkisson P, Weinrich S. Problem behaviour, caregiver reactions, and impact among caregivers of persons with Alzheimer's disease. J Adv Nurs 2001;36:573-582.

3. Yaffe K, Fox P, Newcomer R, et al. Patient and caregiver characteristics and nursing home placement in patients with dementia. JAMA 2002;287:2090-2097.

4. Cummings JL, Mega M, Gray K, et al. The neuropsychiatric inventory: comprehensive assessment of psychopathology in dementia. Neurology 1994;44:2308-2314.

5. Conn D, Thorpe L. Assessment of behavioural and psychological symptoms associated with dementia. Can J Neurol Sci 2007;34(Suppl 1):S67-S71.

6. Kaufer DI, Cummings JL, Ketchel P, et al. Validation of the NPI-Q, a brief clinical form of the neuropsychiatric inventory. J Neuropsychiatry Clin Neurosci 2000;12:233-239.

7. Boada M, Cejudo JC, Tarraga L, Lopez OL, Kaufer D. Neuropsychiatric inventory questionnaire (NPI-Q): Spanish validation of an abridged form of the Neuropsychiatric Inventory (NPI). Neurologia 2002;17(6):317-323.

8. Matsumoto N, Ikeda M, Fukuhara R, et al. Validity and reliability of the Japanese version of the Neuropsychiatric Inventory Caregiver Distress Scale (NPI D) and the Neuropsychiatric Inventory Brief Questionnaire Form (NPI-Q). No To Shinkei 2006;58:785-790.

9. de Jonguhe JF, Kat MG, Kalisvaart CJ, Boelaarts L. Neuropsychiatric inventory questionnaire (NPI-Q): A validity study of the Dutch form. Tijdschr Gerontol Geriatr 2003;34:74-77.
10. American Psychiatric Association Committee on Nomenclature and Statistics. Diagnostic and Statistical Manual of Mental Disorders (DSM-V). Washington, DC: American Psychiatric Association, 2013.

11. McKhann GM, Knopman DS, Chertkow $\mathrm{H}$, et al. The diagnosis of dementia due to Alzheimer's disease: recommendations from the National Institute on Aging-Alzheimer's Association workgroups on diagnostic guidelines for Alzheimer's disease. Alzheimers Dement 2011;7:263-269.

12. Folstein MF, Folstein SE, McHugh PR. "Mini-Mental State": a practical method for grading the cognitive state of patients for the clinician. J Psychiatry Res 1975;12:189-198.

13. Chaves ML, Izquierdo I. Differential diagnosis between dementia and depression: a study of efficiency increment. Acta Neurol Scand 1992;85:378-382.

14. Hughes CP, Berg L, Danziger WL, Coben LA, Martin RL. A new clinical scale for the staging of dementia. $\mathrm{Br} J$ Psychiatry 1982;140:566-572.

15. Maia AL, Godinho C, Ferreira ED, et al. Application of the Brazilian version of the CDR scale in samples of dementia patients. Arq Neuropsiquiatr 2006;64:485-489.

16. Chaves ML, Camozzato A, Godinho C, et al. Validity of the Clinica Dementia Rating Scale for the detection and staging of dementia in Brazilian patients. Alzheimer Dis Assoc Disord 2007;21:210-217.

17. Camozzato AL, Kochhann R, Simeoni C, et al. Reliability of the Brazilian Portuguese version of the Neuropsychiatric Inventory (NPI) for patients with Alzheimer's disease and their caregivers. Int Psychogeriatr 2008;20:383-393. 